\title{
POSTERIOR REVERSIBLE LEUKOENCEPHALOPATHY SYNDROME IN 17 YEAR OLD GIRL
}

\author{
Ana TRIPALO BATOŠ , Goran ROIĆ \\ Department for pediatric radiology \\ Children's Hospital Zagreb, Zagreb \\ Croatia
}

Received: November 11, 2015

Accepted: December 27, 2015

Key words: Posterior leukoencephalopathy syndrome - Magnetic resonance imaging Hypertension.

\begin{abstract}
Objective - To describe a rare condition in children called posterior reversible leukoencephalopathy syndrome, its causes, diagnostic approach and treatment. Case report - A 17 year old girl experienced generalized seizures and head trauma after collapsing in the toilet. It was the first seizure in her life. Five days earlier she had given birth. Two more seizures followed in the next few hours after the first. Blood pressure measured at the time of the seizures was normal, but high afterwards. At that point she was admitted to the intensive care unit. Initially, a computed tomography scan was preformed and it revealed a pathologic finding. A magnetic resonance imaging (MRI) scan was performed to confirm the suspected diagnosis of posterior reversible leukoencephalopathy syndrome (PRES). During her stay in hospital other conditions were excluded and hypertension was considered as the cause of PRES. ACE inhibitors corrected the blood pressure. 12 days later her blood pressure and neurological status normalized, as well as the control MRI finding. Conclusion - Early recognition and intensive care are essential for a completely reversible outcome in children with PRES. In patients with hypertension, cytotoxic agent treatment or corticosteroid treatment, in case of the first seizure in their life, PRES should be suspected. Atypical MRI features can be expected in children, because the anterior watershed region may be as vulnerable as the vertebro-basilar system. In cases of hypertension, the most important issue is hypertension correction; hypertension should therefore be considered and corrected in treating seizures complicated by PRES.
\end{abstract}

\section{Introduction}

Posterior reversible leukoencephalopathy syndrome (PRES) is a clinical-neuroradiological entity (1) characterized by headache, visual disturbances, seizures, altered mental status and radiological findings of edema predominantly in the white matter of the posterior cerebral artery perfusion areas (2). The precise pathogenic mechanisms leading to the development of PRES have not yet been completely identified. Regardless of the triggering factor, breakthrough of autoregulation occurs and causes blood brain barrier disruption primarily at arteriolar level. Therefore, vasogenic edema is presented in the affected areas of the brain $(3,4)$. Because of rare and late appearance of PRES in children compared with adults, pediatricians are less familiar with this condition.

PRES has been reported in different underlying conditions such as renal diseases, autoimmune diseases, pregnancy or cancer 
$(1,5)$. Presumed triggering factors are hypertension, cytotoxic agents or corticosteroid treatment (6). For PRES imaging, magnetic resonance imaging (MRI) $(7,8)$ is the study of choice. Computed tomography (CT) scan findings are often normal and nonspecific, (9) but when positive, hypodense patchy confluent foci are seen, and it is difficult to distinguish PRES from acute stroke. Using the proper MRI protocol (including diffusion weighted images (DWI) and apparent diffusion coefficient (ADC) maps) it is possible to distinguish acute ischemia from the vasogenic edema (1). In literature several different image patterns have been described for PRES lesions $(11,12)$. The common MRI pattern resembles the brain watershed zones, with the cortex as well as subcortical and deep white matter involved to varying degrees. Three hemispheric pattern variants may be involved with similar frequency (holohemispheric, superior frontal sulcal, and primary parietal-occipital). They are usually located in the inferior temporal-occipital junction, superior frontal sulcus, and parietal/occipital region. Partial, asymmetric, or mixed forms of these patterns may be encountered. Focal/ patchy areas of PRES vasogenic edema may also be seen in the basal ganglia, brain stem, and deep white matter (external/internal capsule). Focal areas of restricted diffusion (likely representing infarction or tissue injury with cytotoxic edema) are uncommon (11 to $26 \%)$ and may be associated with an adverse outcome. Hemorrhage (focal hematoma, isolated subarachnoid hemorrhage) is seen in approximately $15 \%$ of patients. (9)

\section{Case report}

A 17 year old girl was admitted to the neuropediatric emergency room from the outpatient clinic, with generalized seizures and head trauma after collapsing in the toilet. It was the first seizure in her life, it lasted for two minutes, during which the patient frothed at the mouth, with eyes wide open. Five days earlier a Caesarean section was performed and the patient gave birth to a healthy baby boy. The second seizure came one hour after the first one and the third followed 5 hours after the second one. The second and the third seizure were stronger than the first one and lasted longer. The patient had mild mental retardation. She had previously suffered head trauma in a car accident and did not regularly perform check-ups with her obstetrician. Blood pressure was measured at the time of seizures and it was normal. The highest value was 140/90 $\mathrm{mmHg}$.

The emergency CT scan in the outpatient clinic revealed hyperdense and hypodense areas in brain parenchyma. Hyperdensities in the left frontal lobe were considered to be traumatic hemorrhagic lesions as the result of the fall in the toilet, and hypodense areas in the white matter of both temporal lobes were considered to be edema. At the time when the girl was admitted to the intensive care unit at our hospital her blood pressure was high $(140 / 100 \mathrm{mmHg})$ and she exhibited left sided hemiparesis and headache. Glasgow Coma Score was 15. Neurologic examination demonstrated no signs of meningism, left sided asymmetry in Migazzini, symmetric reflexes with widening of patellar zone reflex. Patient walked without help, slowly, swinging in the Romberg position. The initial electroencephalogram finding was normal. Because of the patient's lifestyle and living conditions encephalitis was suspected and lumbar puncture was performed. The analysis of cerebrospinal fluid revealed an unspecific finding: glucose level $2.48 \mathrm{mmol} / \mathrm{l}$, chloride level $130 \mathrm{mmol} / \mathrm{l}$, protein level $1,09 \mathrm{~g} / \mathrm{l}$, white blood cells $12 / 3$ per $\mathrm{mm}^{3}$, lymphocytes $9 / 3$ per $\mathrm{mm}^{3}$, erythrocytes $8752 / 3$ per $\mathrm{mm}^{3}$. The elevated proteins and positive erythrocytes finding were considered to be the result of head trauma and the parenchymal hemorrhagic lesion. Visual status and ophthalmoscopy were normal. 24-hour continuous 
monitoring of the patient's blood pressure revealed $18 \%$ systolic and $10 \%$ diastolic values above 95 percentile. She was classified as a non-dipper. Abdominal ultrasound was unremarkable. Electrolytes were unremarkable, with normal values of urea and creatinine.

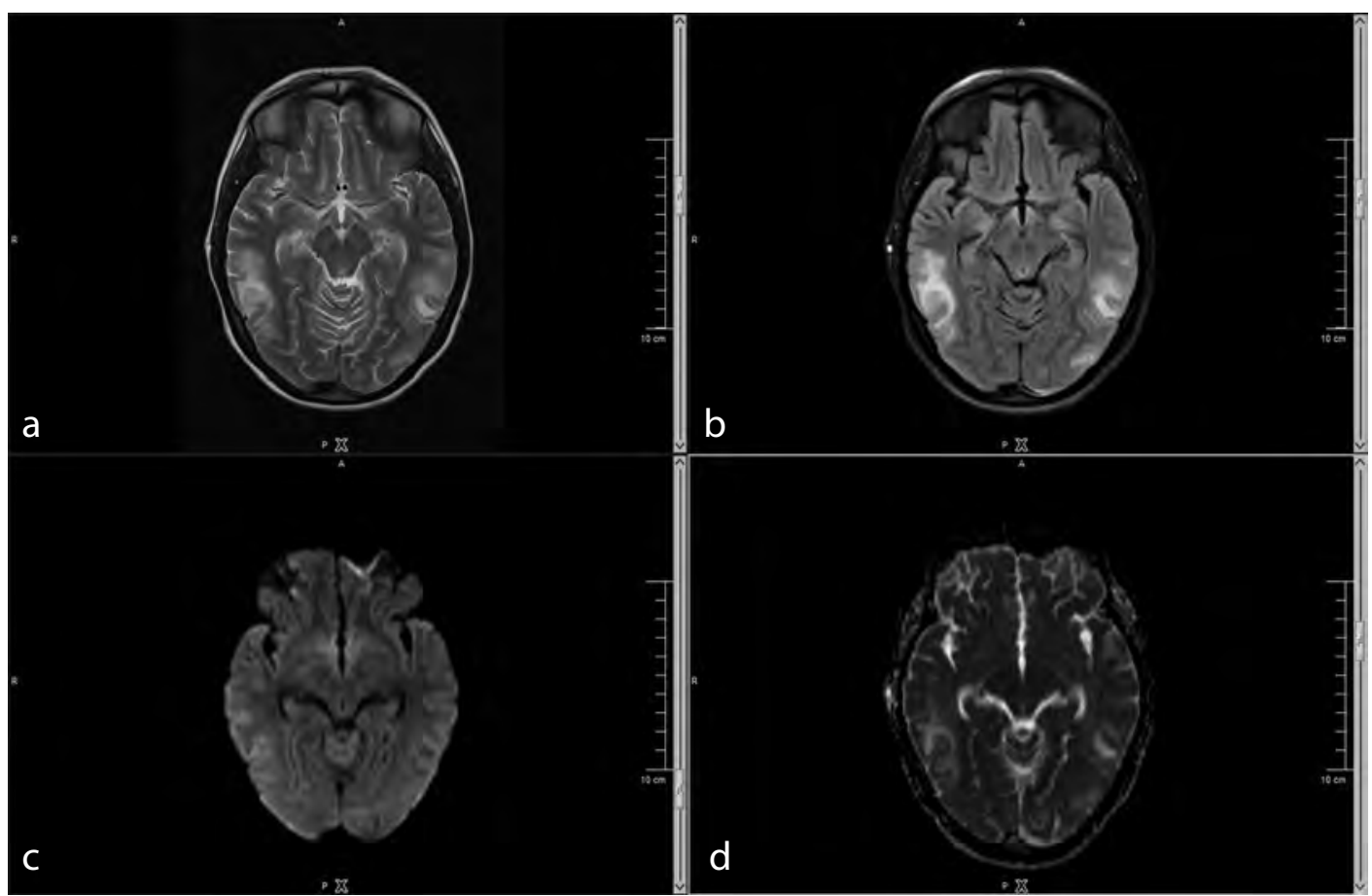

Fig. 1 Initial MRI scan: confluent hyperintense lesions on T2 weighted sequence (a) and FLAIR (b) sequence in subcortical white matter and cortical gray matter of temporal and occipital lobes. DWI (c) and ADC maps (d) of the same area with hyperintense signal. The finding suggests PRES.

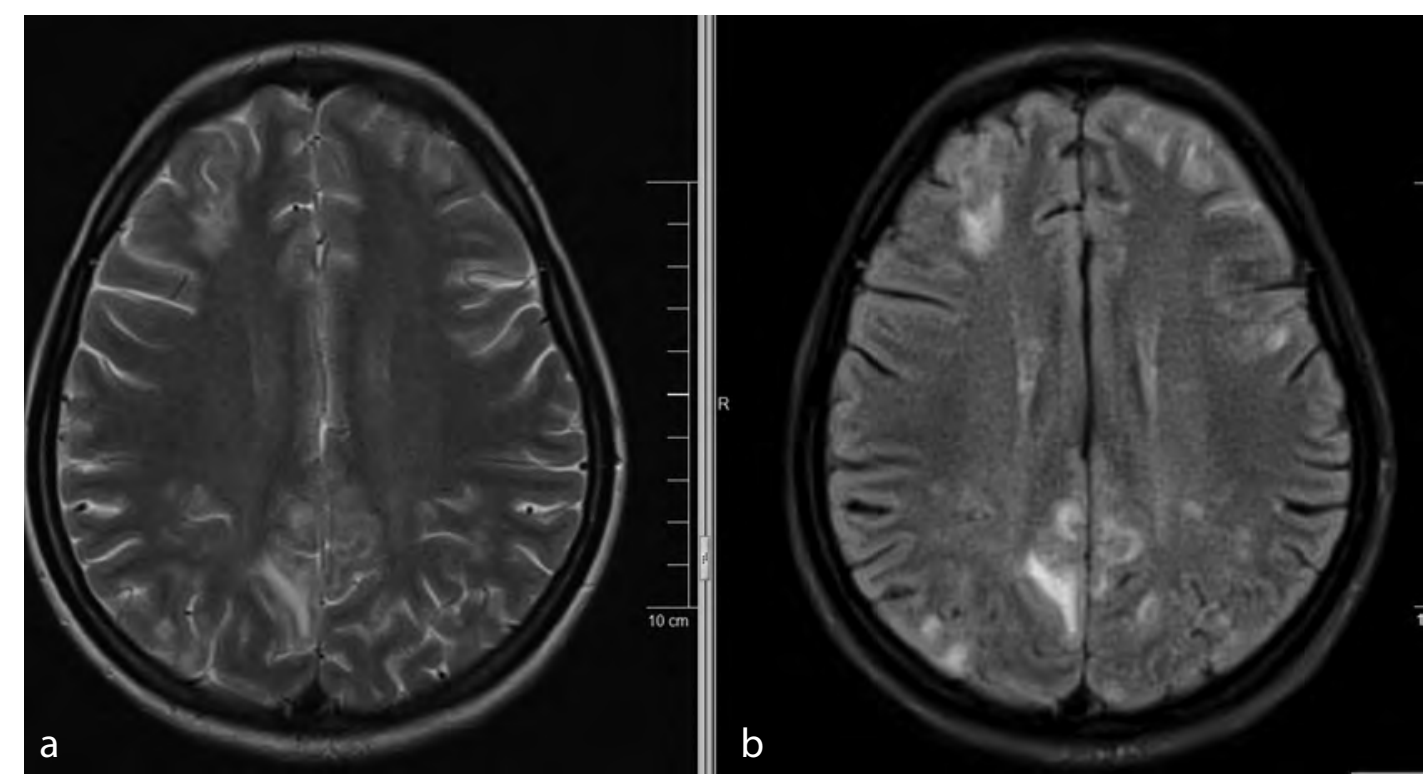

Fig. 2 T2 (a) weighed sequence and FLAIR (b) sequence detect lesions with hyperintensities in subcortical white matter and cortical gray matter of both frontal and parietal regions. 


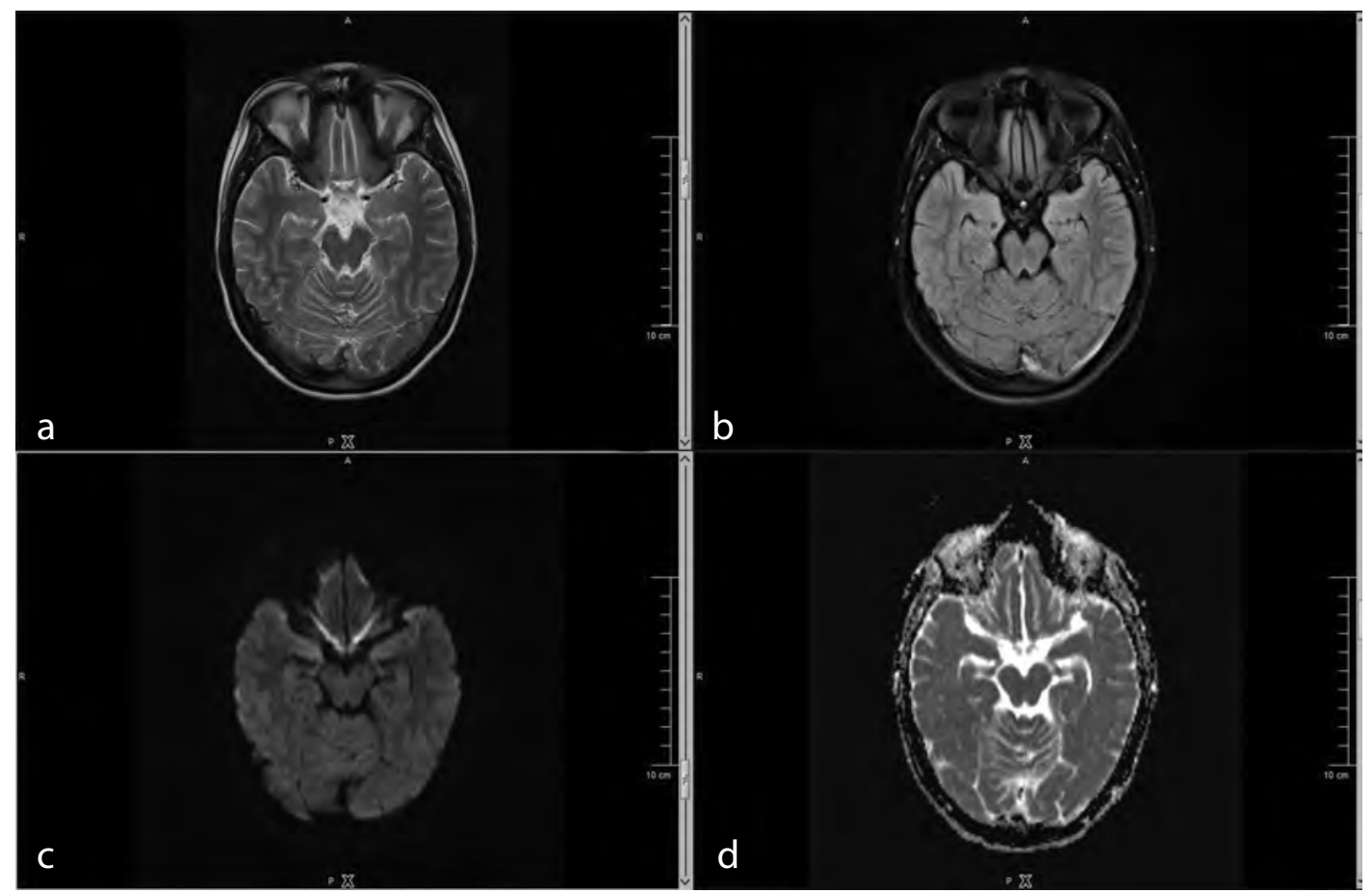

Fig. 3 The control MRI scan detects complete regression of the lesions. T2 weighted sequence (a), FLAIR (b), $D W I$ (c) and ADC maps (d).

Initial therapy included bromocriptine, ACE inhibitor, anticoagulant, barbiturate and intravenous application of $20 \%$ mannitol.

MRI was performed without and with contrast application, as well as 3D time of flight angiography and 2D venography. Exam revealed high signal on T2 weighted and fluid attenuation inversion recovery (FLAIR) sequences bilaterally in the white matter and cortex of the frontal, temporal, occipital and parietal lobes sparing $U$ fibers with patchy enhancement. DWI signal in affected areas was normal with increased signal on ADC maps. The finding corresponded to vasogenic edema in described areas (Fig. 1 and Fig. 2) and PRES was diagnosed. PRES developed as the result of hypertension developed after delivery. 12 days later blood pressure normalized with treatment, as well as the patient's neurological status. Control MRI was performed. Multiple changes were in resolution and the girl was discharged (Fig. 3).

\section{Discussion}

Hypertension is a rare condition in the pediatric population. Therefore, when emergency neurologic pathology is suspected, hypertension is considered as a compensatory response of increased intracranial pressure rather than the cause of acute encephalopathy, and stays unrecognized or is detected late. Some authors suggest the importance of close monitoring of blood pressure in children with critical neurologic illness due to early PRES recognition (6).

When PRES is suspected, early diagnosis is important due to therapeutic implications. The therapeutic approach to PRES considers correction of hypertension. If cerebral acute stroke is diagnosed on MRI the patient might be kept at a higher systemic blood pressure intentionally, to maintain adequate cerebral perfusion $(9,10)$. A prompt distinction between PRES and ischemic stroke, which may overlap, is easy by performing MRI, includ- 
ing DWI and $\mathrm{ADC}$ maps. $\mathrm{DWI}$ and $\mathrm{ADC}$ maps are the sequences of choice to confirm acute stroke (restricted diffusion on DWI and low signal on ADC maps in acute ischemia) and to rule out other conditions that can mimic PRES $(11,13)$. Apart from stroke, differential diagnosis of PRES includes ictal or postictal state, neoplasm, encephalitis, progressive multifocal leukoencephalopathy, inflammatory and infectious processes, mitochondrial encephalomyopathy lactic acidosis and stroke like episodes syndrome, demyelinating pathology and cerebrovascular accidents. The non - specific clinical manifestations and multiplicity of radiological patterns increase diagnostic challenges. The MRI characteristics of these conditions are helpful in diagnosis (9).

\section{Conclusion}

Early recognition and intensive care treatment are essential to a completely reversible outcome in children with PRES. In patients with hypertension, cytotoxic agent treatment or corticosteroid treatment and the first seizure in their life, PRES should be suspected. Atypical MRI features can be expected in children, because the anterior watershed region may be as vulnerable as the vertebro-basilar system. Tai-Heng et al. (6) suggest that features that may be considered atypical in the adult population are actually typical for children with PRES and should potentially be part of the radiologic criteria. In children with hypertension, with the first seizure in their life complicated by PRES, the main task should be hypertension correction.

Authors' contributions: Conception and design: ATB, GR; Acquisition, analysis and interpretation of data: ATB; Drafting the article: ATB, GR; Revising it critically for important intellectual content: ATB, GR.

Conflict of interest: The authors declare that they have no conflict of interest.

\section{References}

1. Hinchey J, Chaves C, Appignani B, Breen J, Pao L, Wang A, et al. A reversible posterior leukoencephalopathy syndrome. N Engl J Med. 1996;334:494500 .

2. Kozak OS, Wijdicks EF, Manno EM, Miley JT, Rabinstein AA. Status epilepticus as initial manifestation of posterior reversible encephalopathy syndrome. Neurology. 2007;69(9):894-7.

3. Doi Y, Kimura F, Fujiyama T, Fujimura C, Nishina T, Sato T, et al. Hypertensive brainstem encephalopathy without parietooccipital lesion--two case reports. Neurol Med Chir (Tokyo). 2006;46:75-9.

4. McKinney AM, Short J, Truwit CL, McKinney ZJ, Kozak OS, SantaCruz KS, et al. Posterior reversible encephalopathy syndrome: incidence of atypical regions of involvement and imaging findings. AJR Am J Roentgenol. 2007;189:904-12.

5. Onder AM, Lopez R, Teomete U, Francoeur D, Bhatia R, Knowbi O, et al. Posterior reversible encephalopathy syndrome in the pediatric renal population. Pediatr Nephrol. 2007;22(11):1921-9.

6. Tai-Heng C, Wei-Chen L, Yong-Hao T, ChienMing T, Tai-Tsung C, Tzeng-Jih L. Posterior Reversible Encephalopathy Syndrome in Children: Case Series and Systematic Review.J Child Neurol. 28(11) 1378-86.

7. Sweany JM, Bartynski WS, Boardman JF. "Recurrent" posterior reversible encephalopathy syndrome: report of 3 cases- -PRES can strike twice! J Comput Assist Tomogr. 2007;31:148-56.

8. Bartynski WS, Boardman JF. Distinct imaging patterns and lesion distribution in posterior reversible encephalopathy syndrome. AJNR Am J Neuroradiol. 2007;28:1320-7.

9. Arzanian MT, Shamsian BS, Karimzadeh P, Kajiyazdi M, Malek F, Hammoud M. Posterior reversible encephalopathy syndrome in pediatric hematologic-oncologic disease: literature review and case presentation. Iran J Child Neurol. 2014;8(2):1-10.

10. Servillo G, Bifulco F, De Robertis E, Piazza O, Striano P, Tortora F, Striano S, Tufano R. Posterior reversible encephalopathy syndrome in intensive care medicine. Intensive Care Med. 2007;33(2):230-6.

11. Covarrubias DJ, Luetmer PH, Campeau NG. Posterior reversible encephalopathy syndrome: prognostic utility of quantitative diffusionweighted MR images. AJNR Am J Neuroradiol. 2002;23(6):1038-48. 
12. Bartynski WS. Posterior reversible encephalopathy syndrome, part 1: fundamental imaging and clinical features. AJNR Am J Neuroradiol. 2008;29(6):1036-42.
13. Tanioka R, Yamamoto Y, Sakai M, Makie T, Mori M, Uehira T, et al. Convalescence of atypical reversible posterior leukoencephalopathy syndrome in human immunodeficiency virus infection. J Med Invest. 2007;54:191-4. 